\title{
Case Report \\ Erosion Infiltration in the Management of Molar-Incisor Hypomineralization (MIH) Defects
}

\author{
Rym Mabrouk $\mathbb{D}^{1},{ }^{1}$ Souha Yahia, ${ }^{2}$ Afef Oueslati, ${ }^{1}$ and Nadia Frih $\mathbb{D}^{3}$ \\ ${ }^{1}$ Endodontics and Restorative Dentistry, Department of Dental Medicine, Hospital of Charles Nicolle, Tunis, Tunisia \\ ${ }^{2}$ Endodontics and Restorative Dentistry, Service of Dental Medicine, Military Principal Hospital of Instruction, Tunis, Tunisia \\ ${ }^{3}$ Forensic Dentistry and Head of Service, Department of Dental Medicine, Hospital of Charles Nicolle, Tunis, Tunisia
}

Correspondence should be addressed to Rym Mabrouk; rim.mabrouk.rm@gmail.com

Received 6 May 2020; Accepted 5 November 2020; Published 11 November 2020

Academic Editor: Yuk Kwan Chen

Copyright (c) 2020 Rym Mabrouk et al. This is an open access article distributed under the Creative Commons Attribution License, which permits unrestricted use, distribution, and reproduction in any medium, provided the original work is properly cited.

White spot lesions caused by enamel demineralization are frequently encountered in dental practice. Their management has always been an important issue in modern dentistry. However, the real dilemma was treating aesthetic demands with noninvasive or minimally invasive techniques preserving the natural tissues. The introduction of resin infiltration technique seems to provide an intermediary treatment modality between prevention and restorative therapy. This case report is aimed at reporting the management of MIH opacities in anterior teeth with resin infiltration technique.

\section{Introduction}

White spot lesions (WSLs), defined as "white opacity," occur as a result of subsurface enamel demineralization that is located on smooth surfaces of teeth [1]. The resulting reduction of the mineral phase compared to healthy enamel leads to intrinsic optical modifications causing white marks. The etiologies are multiple involving preeruptive damages: fluorosis, traumatic hypomineralization, molar-incisor hypomineralization $(\mathrm{MIH})$, or posteruptive lesions: white spots due to carious disease. Among the different clinical presentations of white defects, MIH is the least well-known [2, 3]. Molar-incisor hypomineralization (MIH) is defined as a developmental condition characterized by hypomineralization defects of the enamel on the first permanent molars associated with the permanent incisors. In the literature, the defects have been connected to environmental changes, breast feeding (milk dioxin), respiratory diseases, oxygen shortage of the ameloblasts, and high-fever diseases [4]. The defining clinical features of MIH are demarcated opacities with clear, distinct borders with the adjacent enamel. The opacities range from white to yellow/brown in appearance, and in lesions with a severe mineral deficit, rapid progression to posteruptive enamel breakdown can occur [5].
Incisal enamel defects are frequently quite extensive and most common on the buccal surfaces of the teeth, giving rise to more esthetic concerns [6]. Several techniques have been proposed to improve the appearance of MIH spot lesions. The common treatment strategy comprises restorative procedures, improvement of remineralization using CCP$\mathrm{ACP}$-containing or fluoride-containing products, microabrasion, and bleaching technique laminate veneer restoration. Recently, a resin infiltration technique was introduced with the development of highly flowable resin material [7].

This paper is aimed at describing and illustrating the aesthetic management of white opacities caused by MIH using a resin infiltration technique.

\section{Case Presentation}

A 29-year-old woman was referred to the Department of Dental Medicine of Hospital of Charles Nicolle, Tunis, with the chief complaint of restoring her smile before her wedding soon. The anamnesis did not specify any systemic illnesses of the patient. Intraoral examination revealed white spots in the upper bilateral central and lateral incisors. The spots were easily noticeable in the frontal view of the anterior teeth: big chalky white spots on tooth number 21 and 11 and two 
less pronounced spots on tooth 12 and 22. The spots were well limited, with distinct borders from the adjacent enamel (Figures 1(a) and 1(b)). The periodontal status of the patient was healthy and free from visible plaque, and the radiographic examination revealed no abnormalities of the supporting tissues. After the clinical and radiographic examination, the occurrence of hypomineralization of the upper first molars characterizing MIH confirmed the diagnosis. The erosion infiltration technique, a minimally invasive approach of management of enamel white spots, was proposed and discussed with the consent of the patient. The patient was informed of the infiltration technique's unpredictability, and those possible outcomes ranged from complete disappearance of the lesion to a partial aesthetic improvement. Under her agreement, a decision was made to perform the resin infiltration technique with Icon $^{\circledR}$ (DMG, Hamburg, Germany) on the teeth. Teeth were cleaned with a prophylactic paste at the beginning of the procedure. A rubber dam was applied, and the lesion was locally isolated using a light-cured resin barrier (OpalDam ${ }^{\mathrm{TM}}$ Green Ultradent ${ }^{\circledR}$ ) to ensure a more focalized treatment throughout the different steps of the process (Figure 1(c)). The resin infiltration technique was performed according to the manufacturer's instructions on the upper central and lateral incisors. The enamel surface layer was eroded by the application of $15 \%$ hydrochloric acid gel (Icon-Etch, DMG, Hamburg, Germany) for two minutes (Figure $1(\mathrm{~d})$ ). To achieve a homogeneous etching pattern, the manufacturer's recommendation to stir the gel from time to time, during application, using the smooth surface tips included in the respective product kit was followed. The etching procedure serves to remove superficial discolorations and the higher mineralized surface layer and expose the lesion body. Then, the acid gel was evacuated with a surgical aspirator. Teeth were rinsed for $30 \mathrm{sec}-$ onds using a water spray, and the enamel surfaces were dried. At this time, the lesions were assessed for color modification; no visual color change was obtained. So, the etchant was applied again and reactivated for additional two minutes, until some color alteration could be observed at the wet eroded surface.

Next, the surfaces were air-dried, and ethanol (99\%; Icon-Dry, DMG) was applied for 30 seconds to maximize the water removal inside the lesions (Figure 1(e)). To boost water removal, this step should be repeated at least once. The lesions were air-dried again, and the whitish appearance of enamel lesions was more pronounced (Figures 1(f) and $1(\mathrm{~g}))$.

The resin infiltrant $\left(\operatorname{Icon}^{\circledR}\right)$ was then applied on the lesion surface, and it was allowed to penetrate for three minutes (Figure 1(h)). Excess resin was removed using a blow of air, and light-curing was performed for 40 seconds. The primary result was controlled, and the Icon infiltrant was applied again and allowed to set for one min and then light-cured for $40 \mathrm{~s}$. Finally, the rubber dam was removed. The surfaces were polished using fine-grained abrasive flexible discs, silicone polishers, and finishing strips, depending on the treated area. An immediate esthetic improvement, with total color masking, was observed after treatment (Figure 1(i)).

\section{Discussion}

Enamel developmental defects are caused by various etiological factors, such as amelogenesis imperfecta, fluorosis, trauma hypomineralization, and molar-incisor hypomineralization (MIH). MIH is a qualitative defect of the enamel, characterized by the hypomineralization of one to four permanent first molars, frequently associated with affected incisors. It is also called as hypomineralized permanent first molars (PFMs), idiopathic enamel hypomineralization, nonfluoride hypomineralization, and dysmineralized PFMs [8, 9]. $\mathrm{MIH}$ prevalence varied from $3.6 \%$ to $37.5 \%$ among studies [10]. MIH can sometimes present with opacities in the upper and lower incisors. The characteristic feature of MIH is the clear demarcation between the affected and sound enamels [9]. The incisor defects are usually without loss of enamel substance and are generally less serious than those seen in molars due to the absence of chewing forces [11]. The risk of defects to the upper incisors appears to increase with an increasing number of affected FPMs. Enamel opacities associated with MIH can be problematic for the patients with much esthetic concern. Several techniques have been proposed to improve the appearance of these spots [6]. The described case showed an immediate improvement following the application of the low-viscosity resin, which could be an aesthetic alternative to masking the white opacities caused by molar-incisor hypomineralization [12].

Resin enamel infiltration is described as a microinvasive technology that fills, reinforces, and stabilizes demineralized enamel without drilling or sacrificing healthy tooth structure. This mini-invasive technique can be used to treat smooth surface and proximal carious lesions up to the first third of dentin (D-1) [13]. The procedure for resin infiltration is fairly simple. As the mineralized surface layer of the affected enamel impedes resin from penetrating into the lesion, this layer should be removed using hydrochloric acid gel (15\%) which has been demonstrated to be superior to $37 \%$ phosphoric acid gel in removing the surface layer of natural enamel lesions when applied for 120 seconds. Its penetration depth etching is more than twice $(58 \mu \mathrm{m})$ that of phosphoric acid, enabling penetration into the deepest part of the lesion and eliminating the decalcified areas. The lesion was then desiccated with $99 \%$ ethanol (Icon-Dry) to facilitate the drying process. Icon ${ }^{\circledR}$ works on the principle of infiltration and requires a very dry field. Apart from keeping the environment moisture-free, additional steps must be taken to dry the lesion. This is accomplished by treating the lesion area with alcohol, which evaporates the water within the porosities that could inhibit the process of infiltration $[11,13,14]$.

The last step involved the infiltration of the resin and its light-curing for three minutes. The principle of resin infiltration is to perfuse the porous enamel with resin by capillary action, thereby arresting lesion progression by occluding the microporosities that provide diffusion pathways for the acids and dissolved materials. A positive side effect of resin infiltration is that enamel lesions lose their whitish appearance when these microporosities are filled with the resin. The principle of masking enamel lesions by resin infiltration is based on changes in light scattering within the lesions. 


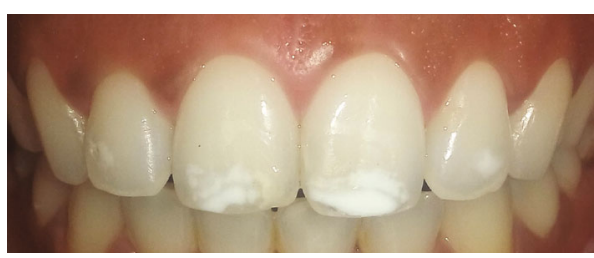

(a)

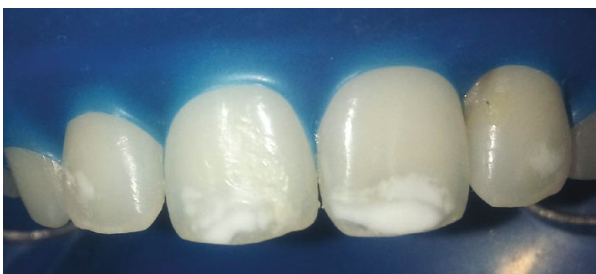

(c)

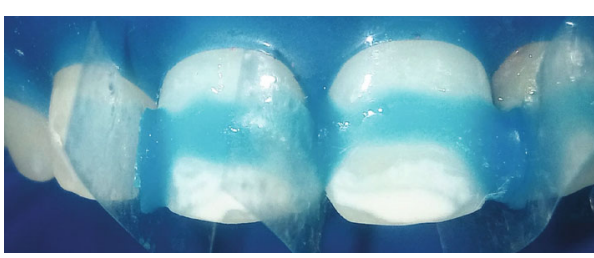

(e)

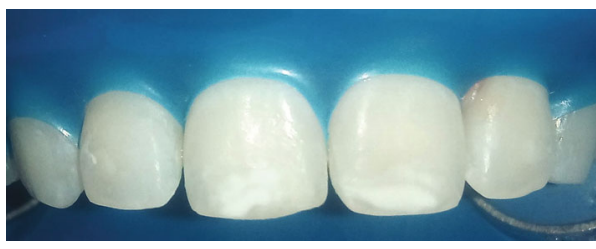

(g)

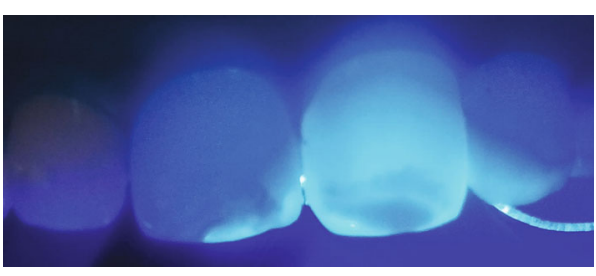

(b)

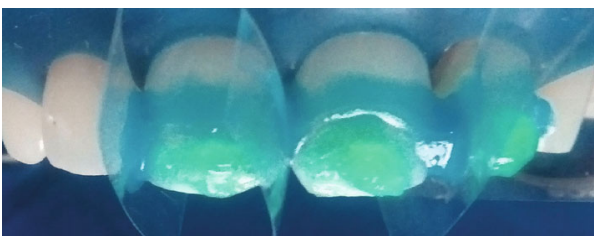

(d)

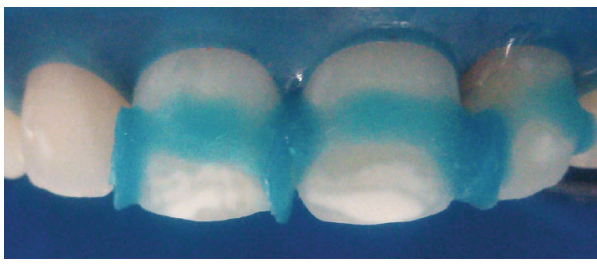

(f)

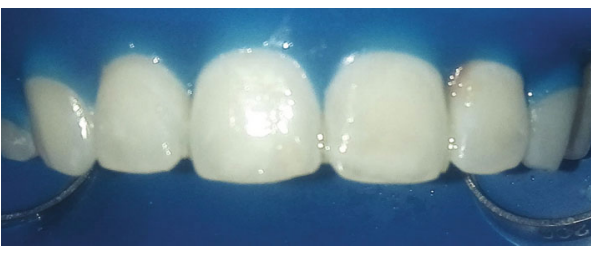

(h)

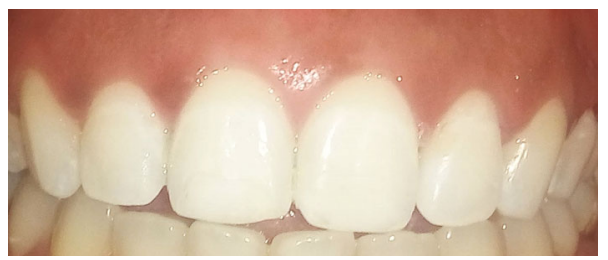

(i)

FIgURE 1: (a) White spot lesion related to molar-incisor hypomineralization. (b) Evaluation of the depth of the lesion under transillumination. (c) Isolation with a rubber dam. (d) Application of the acid gel (120 s) and washing with water spray. (e) Application of an ethanol drier. (f) The appearance of enamel surfaces after ethanol application. (g) Second application of an ethanol drier. (h) Application of a low-viscosity resin infiltration and polymerization. (i) Immediately final result.

Once infiltrated, white spot lesions take on the appearance of the surrounding healthy enamel $[11,15,16]$. In this case report, the resin infiltration technique was applied to the maxillary central and lateral incisors giving an aesthetically satisfactory result, in accordance with the finding of previous studies [8].

Biologically, the resin infiltration technique is a therapeutic of choice to aesthetically manage MIH white enamel spots. In addition to masking enamel opacities, resin infiltration increases the enamel surface hardness improving the mechanical properties of such lesions $[17,18]$. Even though the resin infiltration technique is a noninvasive aesthetic treatment, preserving tooth structure, it still has some limita- tions such as inefficient isolation, incomplete resin polymerization, and the depth of the lesion that may affect the success of the treatment $[16,18]$. Furthermore, organic materials, such as biofilm remnants, carbohydrates, lipids, and proteins, attached to the inner enamel surfaces, could possibly occlude the (underlying) pores in lesions, leading to an incomplete resin penetration of the porous structures and limiting the procedure results.

\section{Conclusion}

White spots caused by molar-incisor hypomineralization could be eradicated using a minimally invasive technique 
which shifts to enamel removal procedures. The patient's motivation would probably play a major role in the success of this approach. This technique is considered to be a microinvasive procedure and might bridge the gap between noninvasive and minimally invasive treatments.

\section{Data Availability}

Data are available from the corresponding author upon request.

\section{Conflicts of Interest}

The authors declare that there is no conflict of interest regarding the publication of this article.

\section{References}

[1] D. Sundararaj, S. Venkatachalapathy, A. Tandon, and A. Pereira, "Critical evaluation of incidence and prevalence of white spot lesions during fixed orthodontic appliance treatment: a meta-analysis," Journal of International Society of Preventive and Community Dentistry, vol. 5, no. 6, pp. 433-439, 2015.

[2] M. Denis, A. Atlan, E. Vennat, G. Tirlet, and J. P. Attal, "White defects on enamel: diagnosis and anatomopathology: two essential factors for proper treatment (part 1)," International Orthodontics, vol. 11, no. 2, pp. 139-165, 2013.

[3] J. P. Attal, A. Atlan, M. Denis, E. Vennat, and G. Tirlet, "White spots on enamel: treatment protocol by superficial or deep infiltration (part 2)," International Orthodontics, vol. 12, no. 1, pp. 1-31, 2014.

[4] K. L. Weerheijm, B. Jälevik, and S. Alaluusua, "Molar-incisor hypomineralisation," Caries Research, vol. 35, no. 5, pp. 390391, 2001.

[5] A. Ghanim, R. Mariño, and D. J. Manton, "Validity and reproducibility testing of the molar incisor hypomineralisation (MIH) index," International Journal of Paediatric Dentistry, vol. 29, no. 1, pp. 6-13, 2019.

[6] S. A. Fayle, "Molar incisor hypomineralisation: restorative management," European Journal of Paediatric Dentistry, vol. 4, no. 3, pp. 121-126, 2003.

[7] S. Jeong-Hye, H. Bock, K. Hyeon-Cheol, and P. Jeong-Kil, "Management of white spots: resin infiltration technique and microabrasion," Journal of Korean Academy of Conservative Dentistry, vol. 36, no. 1, pp. 66-71, 2011.

[8] H. S. Bahadır, G. Karadağ, and Y. Bayraktar, "Minimally invasive approach for improving anterior dental aesthetics: case report with 1-year follow-up," Case Reports in Dentistry, vol. 2018, Article ID 4601795, 5 pages, 2018.

[9] S. Padavala and G. Sukumaran, "Molar incisor hypomineralization and its prevalence," Contemporary Clinical Dentistry, vol. 9, Supplement 2, pp. S246-S250, 2018.

[10] P. Wogelius, D. Haubek, and S. Poulsen, "Prevalence and distribution of demarcated opacities in permanent 1st molars and incisors in 6 to 8 -year-old Danish children," Acta Odontologica Scandinavica, vol. 66, no. 1, pp. 58-64, 2009.

[11] R. Bhandari, S. Thakur, P. Singhal, D. Chauhan, C. Jayam, and T. Jain, "Concealment effect of resin infiltration on incisor of grade I molar incisor hypomineralization patients: an in vivo study," Journal of Conservative Dentistry, vol. 21, no. 4, pp. 450-454, 2018.
[12] K. Srivastava, T. Tikku, R. Khanna, and K. Sachan, "Risk factors and management of white spot lesions in orthodontics," Journal of Orthodontic Science, vol. 2, no. 2, pp. 43-49, 2013.

[13] A. R. Cocco, R. G. Lund, E. N. Torre, and J. Martos, "Treatment of fluorosis spots using a resin infiltration technique: 14-month follow-up," Operative Dentistry, vol. 41-4, pp. 357-362, 2016.

[14] Z. Azizi, "Management of white spot lesions using resin infiltration technique: a review," Open Journal of Dentistry and Oral Medicine, vol. 3, no. 1, pp. 1-6, 2015.

[15] H. Meyer-Lueckel, S. Paris, and A. M. Kielbassa, "Surface layer erosion of natural caries lesions with phosphoric and hydrochloric acid gels in preparation for resin infiltration," Caries Research, vol. 41, pp. 223-230, 2007.

[16] V. Manoharan, S. A. Kumar, S. B. Arumugam, V. Anand, S. Krishnamoorthy, and J. J. Methippara, "Is resin infiltration a micro invasive approach to white lesions of calcified tooth structures?: a systemic review," International Journal of Clinical Pediatric Dentistry, vol. 12, no. 1, pp. 53-58, 2019.

[17] O. Marouane, N. Douki, and F. Chtioui, "A combined approach for the aesthetic management of stained enamel opacities: external bleaching followed by resin infiltration," Case Reports in Dentistry, vol. 2018, Article ID 1605842, 4 pages, 2018.

[18] S. A. Horuztepe, "Effect of resin infiltration on the color and microhardness of bleached white-spot lesions in bovine enamel (an in vitro study)," Journal of Esthetic and Restorative Dentistry, vol. 29, no. 5, pp. 378-385, 2017. 